\title{
Felix Klein: Zur \\ Gründung der \\ IMUK (ICMI) vor \\ einhundert Jahren
}

Gert Schubring

Es ist wohl nicht allgemein bewußt in der mathematischen community, daß Mathematik als Schulfach eine ganz herausgehobene Position einnimmt: es ist das einzige Schulfach, das eine tatsächliche Internationalität praktiziert. Nur in der Mathematik hat es internationale Bewegungen zur Reform des Unterrichts gegeben - und zwar sogar zwei große. Die erste war die internationale Bewegung insbesondere der Jahre 1908 bis 1914 und die zweite praktisch sogar weltweite war mit der sog. modernen Mathematik verknüpft. Darüberhinaus ist die Mathematik auch die einzige Disziplin, für die es eine effektive internationale Organisation gibt und für die regelmäßig umfangreichste internationale Kongresse stattfinden.

Schon 1899 wurde mit L'Enseignement Mathématique die erste internationale Zeitschrift für ein Unterrichtsfach gegründet. Und 1908, in Rom, beim IV. Internationalen Kongress für Mathematik wurde die Internationale Mathematische Unterrichtskommission (Commission Internationale de l'Enseignement Mathématique) gebildet - auf Vorschlag von David Eugene Smith (1860-1944), dem wohl ersten genuinen Mathematik-Didaktiker, nämlich um ,renforcer l'organisation de l'enseignement des mathématiques pures“" (EM 1905, 469).

\section{Zum Mathematikunterricht in Europa bis 1900}

In der Tat war der Stand des Mathematikunterrichts in den europäischen Ländern so tiefgehend unterschiedlich, wie man es sich heute gar nicht mehr vorstellen kann. In Frankreich, das man sich wegen des Ruhms der École Polytechnique gerne als Land mit starkem Mathematikunterricht vorstellt, war bald nach der Restaura- tion des Königtums I8I5 praktisch eine Rückkehr zum Modell der Jesuiten-collèges erfolgt: nach etwas Rechenunterricht in den zwei Anfangsklassen gab es erst in der Abschlussklasse Unterricht in Mathematik. Erst ab 1885 gab es in jeder Klasse solchen Unterricht, zumeist aber nur in einer oder zwei Wochenstunden. In Italien, das ab 1859 zu einem Nationalstaat vereinigt wurde, wurde die Mathematik in die klassische Konzeption der Gymnasien eingefügt: als geistbildendes Fach und mit Euklid als offiziellem Lehrbuch. Die ständigen Misserfolge der Schüler in den zentralen Abschlussprüfungen gegenüber diesen Anforderungen führten zu einer in Europa einmaligen Entwicklung: die Mathematik wurde von den Abschlussprüfungen in den I880ern ausgenommen und war damit nicht mehr Hauptfach.

In England war die Situation in mancher Hinsicht analog zu Italien. Hier hatten sich erst im Laufe des 19. Jahrhunderts einige Sekundarschulen gebildet und damit begonnen, das Schulsystem zu differenzieren vom vorherigen allein dominierenden college-System der englischen Universitäten. Strikt und dogmatisch befolgtes Lehrbuch war auch hier Euklid, als Mittel der Ausbildung logischen Denkens. Seit den I870er Jahren gab es hier Reformansätze, um die Dominanz von Euklid als Lehrbuch zu brechen.

In Deutschland war die Situation ganz disparat. Die seit $1815 \mathrm{im}$ deutschen Bereich bestehenden 39 Staaten und Stadtstaaten entwickelten sich nicht nur politisch und sozial unterschiedlich, sondern auch bildungspolitisch. Die Mathematik war daher in den höheren Schulen dieser Staaten gleichfalls unterschiedlich institutionalisiert. Diese Situation änderte sich nicht mit der Gründung des Kaiserreichs aus den - nach den Annexionen durch 
Preußen 1866 - nunmehr 25 kleindeutschen Staaten, d. h. ohne Österreich. Einen Eindruck von der Spannweite des bestehenden Spektrums bildet der Vergleich von Preußen, mit seinem gut etablierten Mathematikunterricht, mit dem Königreich Württemberg. In Württemberg hat sich die Säkularisierung des Bildungssystems besonders spät vollzogen. Das findet seinen charakteristischen Ausdruck darin, daß der Unterrichtsstoff an den Gymnasien und an den ihnen entsprechenden Lateinschulen bis zum Ende der Mittelstufe von der Vorbereitung auf das sogenannte Landexamen bestimmt war: der Aufnahmeprüfung für „evangelischtheologische Seminare", den früheren Klosterschulen, die auf ein Studium der Theologie vorbereiteten. Da dies noch die vorherrschende Berufsorientierung für Schüler der höheren Schulen war, wurden Gegenstände, die nicht im Landexamen geprüft wurden, im Gymnasium vernachlässigt. So kam es in Württemberg zu der Sondersituation, daß - wie Jahrhunderte zuvor - nur wenige, auch für Theologen für relevant gehaltene Arithmetik gelehrt wurde, die in anderen Staaten eher dominierende Geometrie aber fast ganz fehlte. Die Mathematik konnte so nicht den Charakter eines Hauptfaches innehaben.

$\mathrm{Da}$ das Kaiserreich lediglich ein Staatenbund war, in dem insbesondere jeder Staat die Hoheit für sein Bildungswesen behielt, war zunächst keine Änderung dieser unterschiedlichen Strukturen abzusehen, obwohl PreuBen naturgemäß eine dominierende Position einnahm, da es knapp zwei Drittel sowohl der Fläche wie der Bevölkerung beherrschte. Das zweite wesentliche Merkmal für die Geschichte des Mathematikunterrichts war, daß in Deutschland noch in einer weiteren Hinsicht sich ein Sonderweg durchgesetzt hatte: die Aufspaltung des höheren Schulwesens in unterschiedlich profilierte und sozial bewertete Schultypen. In Preußen hatte Wilhelm von Humboldt (1767-1835) das Gymnasium als gemeinsame höhere Schule geplant, in dem nicht nur studierwillige, sondern auch bürgerliche Berufe anstrebende Schüler unterrichtet werden sollten. Gleichwohl wurden gegen die Mitte des 19. Jahrhunderts von Kommunen zunehmend für diese Schülergruppe Realschulen gegründet, deren Struktur auch lehrplanmäBig schließlich 1859 staatlich geregelt wurde. Während aber in Preußen Gymnasien und Realschulen in ihren Lehrplänen nicht komplementär waren, d.h. es gab sowohl alte Sprachen an Realschulen als auch Mathematik und Naturwissenschaften an Gymnasien, wurden in anderen deutschen Staaten Realschulen gegründet, um die Marginalisierung von Mathematik und Naturwissenschaften an den klassisch

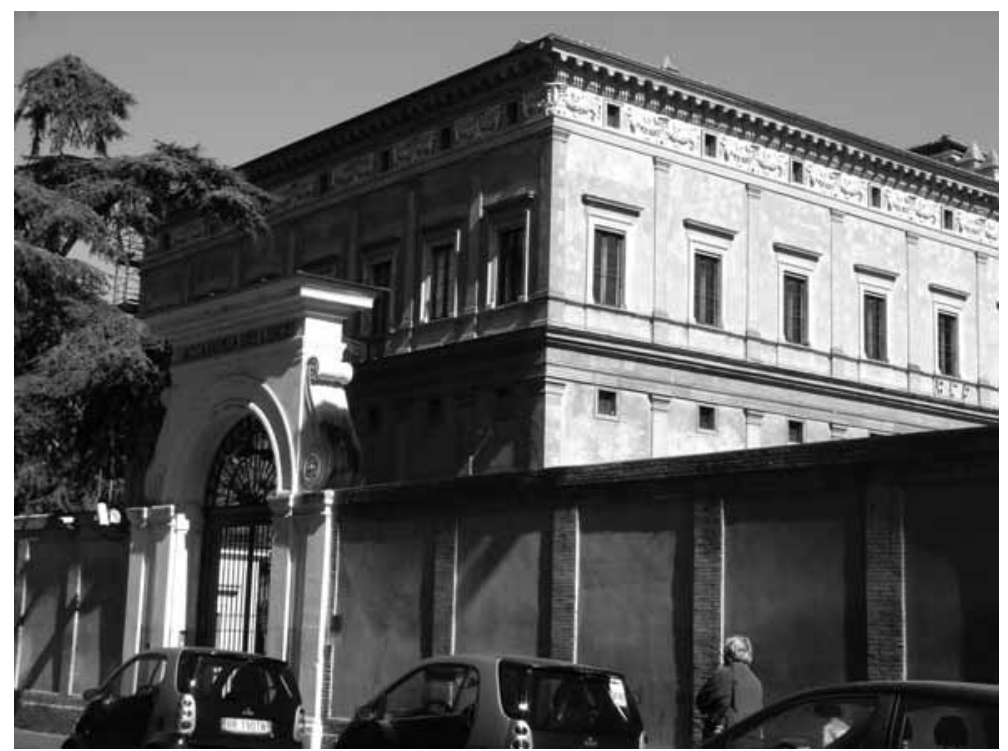

Die Accademia dei Lincei, Ort des ICM 1908

ausgerichteten Gymnasien auszugleichen. So ist die auch im Namen zum Ausdruck kommende Spaltung der Bildungskonzeption - das Gegenüber von „humanistischem“ Gymnasium und Realgymnasium - zuerst in Bayern eingeführt worden, im Jahre 1854 bzw. I 864 [vgl. Schubring, 1989a].

Die Parallelität von schließlich drei Typen höherer Schulen mit Abiturberechtigung, aber mit unterschiedlich institutionalisierten Bildungskonzeptionen - den Gymnasien, den Realgymnasien und den Oberrealschulen - war in anderen europäischen Staaten nicht anzutreffen.

Die Situation des Mathematikunterrichts an den höheren Schulen in allen Staaten des Deutschen Reiches war gegen Ende des 19. Jahrhunderts von dem Widerspruch gekennzeichnet, daß die Unterrichtsziele elementarisch ausgelegt waren, mit dem Schwerpunkt auf der klassischen Geometrie, um dem Bildungsziel des logischen Denkens zu genügen, während zugleich die rasche industrielle Entwicklung eine moderne wissenschaftlich-technische Grundbildung erforderte. Die Behandlung von Variablen und damit auch von Funktionen war ausgeschlossen. Entsprechend wurden Kegelschnitte nur synthetisch, über geometrische Örter, behandelt und nicht analytisch - analytische Geometrie war ebenso ausgeschlossen.

\section{Die Reforminitiativen von Klein}

Initiativen für Reformen gingen allerdings nicht von den Mathematiklehrern selbst aus, sondern von einem weitblickenden Mathematiker, von Felix Klein (vgl. Schubring 1989). Klein hatte am deutlichsten gespürt, daß der Mathematikunterricht sich in einer tiefen Krise befand und 


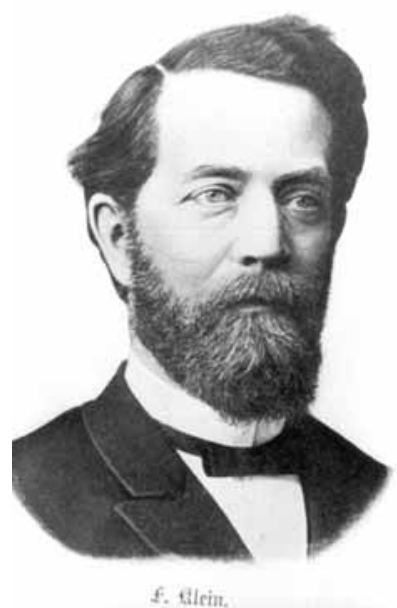

hatte seit den 1890er Jahren begonnen, sich insbesondere der Verbesserung der Lehrerausbildung zu widmen - als einem ersten Schritt, um die von inm beklagte „doppelte Kluft“ zwischen Schulmathematik und Hochschulmathematik zu überbrücken, die „Diskontinuität“" mit ihrem „System des doppelten Vergessens“ zu beseitigen.

Den entscheidenden Anstoß, eine Agenda für die Reform mathematischer Lehre im Systemzusammenhang von höherer Schule, Hochschule und Lehrerbildung zu entwickeln, bildete schließlich der Auftrag des preußischen Kultusministers an Klein zu einem Gutachten für die große Schulkonferenz 1900, und zwar einerseits über die weitere Entwicklung des mathematischen und naturwissenschaftlichen Unterrichts und andererseits darüber, welche Art der Vorbildung an höheren Schulen für das Studium an Technischen Hochschulen vorzuziehen sei.

Kleins Gutachten vom Mai 1900 bildet ein Schlüssel-Dokument für die Geschichte der mathematischen Unterrichtsreform.' Es enthält sein Programm zur Neustrukturierung des Verhältnisses von Sekundar- und Hochschulbildung, und es beruht auf den Konsequenzen, die er aus der Antimathematiker-Bewegung der 1890er Jahre unter den Ingenieuren gezogen hat. Der Hauptpunkt in Kleins Antwort auf die erste Frage war, daß alle drei Typen höherer Schulen hinreichende Möglichkeiten für einen erfolgreichen Mathematikunterricht boten. Für die Antwort zur zweiten Frage beschrieb er die tiefe Krise, in der sich die mathematische Lehre an den Technischen Hochschulen befand. Ursprünglich hatte dort die Mathematik, gemäß deren berühmtem Modell, der École Polytechnique, das Hauptelement der allgemeinen Studien aller Studenten gebildet, das sie abzuschließen hatten, bevor sie das Studium in speziellen technischen Fächern beginnen konnten. Diese polytechnische Funktion der Mathematik war aber schon lange obsolet geworden. Mathematische Forschung und Lehre hatten sich immer mehr zur Grundlagen-Seite hin entwickelt, wie es im Programm der Arithmetisierung der Analysis zum Ausdruck kam. Andererseits hatten sich die technischen Disziplinen hochgradig spezialisiert und ihren Charakter verändert; sie hatten kein Interesse mehr an den komplexen Fragestellungen der höheren Mathematik und bevorzugten vielmehr die „geniale Intuition“. Die Folgen dieser Orientierungsänderung für die Mathematik an den Technischen Hochschulen beschrieb Klein als fatal: die Mehrheit von deren Studenten weigerten sich schlicht, den mathematischen Vorlesungen zu folgen und schwänzten sie. Und die Mathematik-Dozenten erhielten keine Unterstützung von ihren Ingenieur-Kollegen.

Klein schlug in seinem Gutachten eine radikale Problemlösung vor. Da die Mathematik Vorlesungen an den Technischen Hochschulen aus einem vorbereitendem allgemeinen Teil und einem fortgeschrittenen oder höheren Teil bestanden, empfahl er, daß der Grundlagenteil in die Vorbereitungs-, d. h. in die höheren Schulen verlegt werde und daß nur der fortgeschrittene Teil weiter an den Technischen Hochschulen gelehrt werde - aber in reformierter Weise: nicht mehr als unabhängige mathematische Disziplinen, sondern in ständiger Abstimmung mit den Erfordernissen des Studiums für Ingenieurberufe. Und als solche Grundlagenfächer, die an den Mathematikunterricht der höheren Schulen übergehen sollten, bestimmte Klein die analytische Geometrie und die Differenzial- und Integralrechnung.

Natürlich betraf der schwierigste Teil seiner Neustrukturierungsvorschläge die Schulmathematik. In den Jahren 1900 und 190I verhandelte Klein mit dem preußischen Kultusministerium, um es zu überzeugen, die Reformen in traditioneller Weise als neuen Lehrplan anzuordnen. Die schließliche Antwort vom April 1902 war unerwartet und ganz außergewöhnlich. Obwohl das Ministerium den Vorschlägen zustimmte, lehnte es es ab, sie als Lehrplanänderungen von oben her vorzuschreiben. Es empfahl ihm vielmehr, die Einführung der curricularen Veränderungen von unten her zu organisieren: durch Gewinnung der Unterstützung von geeignet ausgebildeten Lehrern, die als Promotoren der Implementation der Reformen an ausgewählten Schulen agieren würden. Indem das Ministerium weiterhin am neuhumanistischen Grundsatz der methodischen Lehrfreiheit der Gymnasiallehrer (vgl. Schubring 1983, Kap. I3) festhielt, konnten diese Lehrer Kleins Curriculumänderungen in die Praxis umsetzen ohne vorherige behördliche Genehmigung.

In der Tat gelang es Klein, mittels des von ihm eingeführten Schlüsselbegriffs des funktionalen Denkens eine breite Bewegung zu initiieren, ausgehend von früheren Schülern unter den Mathematiklehrern, aber darüber hinaus viele weitere Lehrer und dann auch Lehrerverbände erfassend. Die Reformlehrer praktizierten nicht nur einen modernisierten Unterricht in Arithmetik, Algebra und Geometrie auf der Grundlage des funktionalen Denkens und der Betonung der Raum-Anschauung, sondern sie führten auch die Anfangsgründe der Differenzialund Integralrechnung ein.

Für Kleins Programm der flexiblen Übergänge von allen drei Typen höherer Schulen in al-

I Kleins Konzeptfassung ist abgedruckt in: Schubring 1989, 212-220, und die endgültige Fassung in: Schubring 2000, 69-74. 
le Hochschul-Studiengänge war es wesentlich, daß die Curriculumreform nicht auf die beiden Realanstalten - das Realgymnasium und die Oberrealschule - beschränkt blieb, sondern ebenso auch das Gymnasium umfasste. Dagegen mobilisierten sich aber noch für längere Zeit Widerstände. 1904 hatte die Gesellschaft Deutscher Naturforscher und Ärzte (GDNÄ) auf Vorschlag von Klein eine Unterrichtskommission gebildet, um konsensfähige Reformvorschläge für den mathematischen und naturwissenschaftlichen Unterricht - die sogenannte Breslauer Kommission - auszuarbeiten, um so mit einem Curriculumvorschlag für Mathematik und Naturwissenschaften zusammen der Reformbewegung eine noch breitere Plattform zu geben. Das Kommissionsergebnis, die sogenannte Meraner Reform oder das Meraner Programm - da es 1905 bei der Jahrestagung der GDNÄ und DMV in Meran vorgestellt wurde $^{2}$-, entsprach für die Mathematik weitgehend dem Kleinschen Konzept, enthielt aber den Wermutstropfen, daß die Elemente der Differenzial- und Integralrechnung gerade für das Gymnasium nicht vorgesehen waren, sondern daß deren Einführung der Initiative einzelner Lehrer überlassen worden war.

\section{Ablauf der Beratungen in Meran}

Was ist nun tatsächlich in Meran geschehen? Der Bericht von Krazer, dem Schriftührer der DMV im Jahresbericht der DMV ist von lakonischer Kürze:

Hierauf [nach den Formalien] war die Gesamtsitzung der beiden wissenschaftlichen Hauptgruppen, in welcher zum Beginn Gutzmer - Halle a. S. als Vorsitzender der Unterrichtskommission einen Bericht über deren bisherige Tätigkeit erstattete. (Krazer 1905, 5I8).

Dieser Kurzbericht zeigt, daß in Meran lediglich ein Bericht erstattet und keine Beschlüsse über den Meraner Lehrplan gefaßt wurden. Andererseits zeigt der Bericht über die gleiche Versammlung in den Unterrichtsblättern für Mathematik und Naturwissenschaften - der zu der ZfmnU parallelen Zeitschrift -, daß zugleich aber eine ganz eindeutige Meinungsbildung und -äußerung stattgefunden hat.

Der Bericht des Herrn Gutzmer über die Tätigkeit der „Unterrichtskommission“ wurde von der sehr zahlreich besuchten Versammlung mit grösster Aufmerksamkeit entgegengenommen, verschiedentlich von spontanem Beifall unterbrochen und zum Schluss mit sehr lebhafter Zustimmung begrüsst. Der Bericht war den Be- suchern der Versammlung schon gedruckt zugänglich und ihm lagen die von der Kommission ausgearbeiteten Vorschläge zu Lehrplänen in Mathematik, Physik, Chemie und Biologie bei. Obwohl für eine grosse Zahl von Exemplaren gesorgt war, reichten sie bei weitem nicht aus: auch ein Zeichen dafür, in welchem Masse der Vortrag des Vorsitzenden der Unterrichtskommission das Interesse der weitesten Kreise zu erwecken verstanden hatte. [...] Es liegt in der Natur der behandelten Materie, daß eine sofort sich anknüpfende Diskussion ohne ein genaueres Studium der vorgelegten Lehrpläne keinen besonderen Wert hätte beanspruchen können; es ist daher nur mit Dank zu begrüssen, wenn der Vorsitzende der Gesellschaft bei der Eröffnung der Diskussion die Bitte aussprach, nur allgemeine Wünsche und Zusätze vorzubringen: dagegen zu vermeiden, in ausführlicher Diskussion auf einzelne Thesen oder sonstige Details näher einzugehen, da doch ein bestimmtes Ziel nicht erreicht werden könnte. Diesem Wunsche wurde auch seitens der Versammlung entsprochen; die Diskussion beschränkte sich auf die Anregung des Herrn Professors Dr. Herz - Wien, eine analoge Kommission auch für Oesterreich einzusetzen. Dieser Antrag wurde aber, nachdem Professor Gutzmer und Professor Czuber - Wien dagegen sich ausgesprochen, von der Versammlung abgelehnt (Schotten 1906, 39 f.).

Zur weiteren expliziten Meinungsäußerung über die Reform kam es danach in zwei Vorträgen. Die zwei Hauptvorträge in der Abteilung für Mathematik behandelten beide die Einführung der Infinitesimalrechnung in den Schulunterricht und bildeten intensive Plädoyers für die Reformziele des Meraner Programms, für das funktionale Denken als Grundelement des mathematischen Unterrichts und für die Einführung der Infinitesimalrechnung an den höheren Schulen - in Süddeutschland und Österreich ,Mittelschulen' genannt - als Konsequenz. Und die Teilnehmer stimmten diesen Forderungen auch ausdrücklich zu:

An den Vortrag knüpfte sich eine sehr lebhafte Diskussion, in der allgemein zum Ausdruck kam, dass die Abteilung den Gedanken des Vortragenden durchaus zustimmte und für die Einführung der Elemente der Infinitesimalrechnung in den Lehrplan der Mittelschulen ist (ibid., 40).

2 Das „Meraner“ Programm ist publiziert in: Zeitschrift für mathematischen und naturwissenschaftlichen Unterricht, 36 (1905), 533-557. 


\section{Die Arbeiten der IMUK}

Beim Internationalen Mathematiker-Kongreß in Rom war ein „Comité Central“ für die IMUK gewählt worden, bestehend aus Felix Klein, Henri Fehr (Schweiz) und George Greenhill (Großbritannien). Es wählte Klein zum Präsidenten. Klein entwickelte auch in diesem Feld eine umfassende und dynamische sowohl organisatorische wie konzeptionelle Aktivität. Einerseits weitete er gegenüber der vom Kongreß vorgesehenen recht restriktiven Einladung von teilnehmenden Staaten diese auf eine wesentlich größere Zahl von Staaten auf allen Kontinenten aus. Andererseits bezog er statt der vom Kongreß nur intendierten Sekundarschulen - sämtliche Typen von Schulen ein: sowohl allgemeinbildende als auch berufsbildende. Und gegenüber der ursprünglichen berichterstattend-deskriptiven Aufgabenstellung erweiterte er diese auch auf die Förderung von Reformen des Mathematikunterrichts (s. Schubring 2003, 55 ff.). Ein Hauptmittel zu dieser Förderung bildeten die vergleichenden internationalen Untersuchungen zu Kernproblemen des Mathematikunterrichts. Insgesamt wurden bis 1914 acht solcher Analysen in Angriff genommen und bei internationalen IMUKTagungen oder beim nächsten MathematikKongreß vorgestellt und beraten:

I. La fusion des différentes branches mathématiques dans l'enseignement dans les écoles moyennes. (Mailand I9II)

2. La rigueur dans l'enseignement mathématique dans les écoles moyennes (Mailand I9II).

3. L'enseignement mathématique théorique et pratique destiné aux étudiants en sciences physiques et naturelles. (Mailand I9II)

4. La préparation mathématique des physiciens à l'université. (Cambridge 19/2)

5. L'intuition et l'expérience dans l'enseignement mathématique dans les écoles moyennes. (Cambridge 1912)

6. Les résultats obtenus dans l'introduction du calcul différentiel et intégral dans les classes supérieures des établissements secondaires. (Paris 1914)

7. La préparation mathématique des ingénieurs dans les différents pays. (Paris 1914)

8. La formation des enseignants (professeurs) des mathématiques pour les établissements secondaires.

Den Höhepunkt in den Tätigkeiten der IMUK bildete ihr Kongreß 1914 in Paris. Die dort vorgestellten und diskutierten Berichte entsprachen den zwei Kernpunkten in Kleins ReformAgenda: der mathematischen Ausbildung der Ingenieure und die bisherigen Ergebnisse der Einführung der Elemente der Infinitesimalrechnung in die Schulen. Gerade der zweite Bericht war am sorgfältigsten von Klein selbst vorberei- tet worden; er hatte nicht nur den internationalen Fragebogen ausgearbeitet, der Grundlage der Analyse wurde, sondern auch den Berichterstatter ausgesucht: Emanuel Beke, einen ungarischen Mathematiker und früheren Schüler. Ein besonders eindrückliches Dokument für Kleins Engagement für die Reform des Mathematikunterrichts stellt die Reihe Abhandlungen über den mathematischen Unterricht in Deutschland dar, die die Berichte der deutschen IMUKKommission an die IMUK bilden und die in 47 einzelnen Abhandlungen eine klassische und sowohl umfassende wie detaillierte Analyse des Zustands des mathematischen Unterrichts in den verschiedenen Schultypen der deutschen Staaten geben. Klein war unermüdlich tätig, die Berichte organisatorisch und konzeptionell anzuregen, zu betreuen und ihre Fertigstellung sicherzustellen. Besonders am Herzen lag ihm Wilhelm Loreys Abhandlung zur Mathematik an den deutschen Universitäten.

Es ist auch nur wenig bekannt, daß der ICM in Rom nicht eine permanente Kommission gegründet hatte; die IMUK hatte vielmehr für ihre berichtende Aufgabe ein Mandat von vier Jahren erhalten, bis zum nächsten Kongreß: 1912 in Cambridge. Aufgrund der Ausweitung der Aufgaben durch Klein waren bis 1912 die Arbeiten jedoch noch nicht beendet und so verlängerte der Kongreß das Mandat um weitere vier Jahre, bis zum Kongreß 1916. Die IMUKTagung in Paris war jedoch für lange Zeit die letzte tatsächlich internationale Tagung. Nur kurz danach brach der Erste Weltkrieg aus. Klein hoffte, die Arbeiten der IMUK fortführen zu können und trieb die Autoren der noch ausstehenden deutschen Berichte zur Fertigstellung an. Doch seine Unterschrift im Oktober 1914 unter den unsäglich chauvinistischen „Aufruf an die Kulturwelt" führte nicht nur zu seinem Ausschluß aus der Pariser Akademie, sondern der Aufruf bewirkte den Boykott der Kooperation mit deutschen Wissenschaftlern durch die Alliierten. Die Arbeit der IMUK kam zum Stillstand und I 920 mußte Klein die Auflösung der IMUK zur Kenntnis nehmen.

Bis zu dieser Auflösung waren 294 Berichte und Abhandlungen, aus 17 Ländern, publiziert worden. Sowohl in Hinsicht von Publikationen als auch in tatsächlicher Veränderung von Mathematikunterricht war die Wirkung der IMUK und der ersten internationalen Reformbewegung enorm. Die deutsche IMUK-Kommission hat ihre Arbeiten fortgesetzt; Klein hatte den Vorsitz an Walther Lietzmann weitergegeben. Lietzmann blieb Vorsitzender bis nach dem zweiten Weltkrieg, als schließlich Heinrich Behnke den Vorsitz übernahm. Auf dem Mathematiker-Kongreß 1928 in Bologna, dem ersten, an dem deutsche Mathematiker wieder eingeladen waren und teilnahmen, wurde auch die Arbeit der IMUK wieder 
aufgenommen, allerdings auf viel weniger aktivem Niveau als ursprünglich. Auch jetzt waren es jeweils vierjährige Mandate, die jedoch beim nächsten ICM verlängert wurden. Erst nach dem Zweiten Weltkrieg, 1952, wurde die IMUK neu konstituiert, nunmehr als ICMI - International Commission on Mathematics Instruction - und mit nicht mehr befristetem Auftrag. Die grundlegende Bedeutung des Wirkens von Felix Klein für den Mathematikunterricht ist im Jahre 2002 von der ICMI durch die Einrichtung der Felix Klein Medal gewürdigt worden. Sie wird verliehen an Wissenschaftler für deren Lebenswerk für die Mathematik-Didaktik. Sie wurde zum ersten Mal beim Internationalen Mathematikunterrichts-Kongreß im Jahre 2004 verliehen, an Guy Brousseau. Im März 2008 wird in Rom ein Symposium zur Hundertjahrfeier der Gründung der IMUK/ICMI stattfinden, in der gleichen Accademia dei Lincei, in der 1908 der ICM getagt hatte. Und im September 2008 wird in Erlangen im Rahmen der Jahrestagung der Deutschen Mathematiker-Vereinigung ein Mini-Symposium zu Felix Klein und 100 Jahre IMUK stattfinden.

\section{Bibliographie}

Abhandlungen über den mathematischen Unterricht in Deutschland. Veranlasst durch die Internationale Mathematische Unterrichtskommission, hrsg. v. Felix Klein. B. G. Teubner: Leipzig und Berlin, Bände I bis V, 1909 bis 1916.

Geck, Erwin: Der mathematische Unterricht an den höheren Schulen: nach Organisation, Lehrstoff $u$. Lehrverfahren u. Ausbildung der Lehramtskandidaten im Königreich Württemberg. B. G. Teubner: Leipzig und Berlin, 1910.

Carl Krazer, „Bericht über die Jahresversammlung in Meran vom 24. bis 29. September 1905“, Jahresbericht der Deutschen Mathematikervereinigung, 1905, 14: 516519.

Heinrich Schotten, ,Bericht über die 77. Versammlung Deutscher Naturforscher und Aerzte in Meran“, Unterrichtsblätter für Mathematik und Naturwissenschaften, 1906, 12, 2: 39-41.

Schubring, Gert: Die Entstehung des Mathematiklehrerberufs im 19. Jahrhundert: Studien und Materialien zum Prozeß der Professionalisierung in Preußen (I8/0-1870). Beltz: Weinheim 1983.
Schubring, Gert: „Pure and Applied Mathematics in Divergent Institutional Settings in Germany: the Role and Impact of Felix Klein", The History of Modern Mathematics. Volume II: Institutions and Applications eds. David Rowe, John McCleary (Academic Press: Boston 1989), S. I7|-220.

Schubring, Gert: „Die Mathematik - ein Hauptfach in der Auseinandersetzung zwischen Gymnasien und Realschulen in den deutschen Staaten des 19. Jahrhunderts", Bildung, Staat und Gesellschaft im 19. Jahrhundert. Mobilisierung und Disziplinierung. Hrsg. K.-E. Jeismann. F. Steiner: Stuttgart 1989, S. 276-289. [1989a]

Schubring, Gert: „Felix Kleins Gutachten zur Schulkonferenz 1900: Initiativen für den Systemzusammenhang von Schule und Hochschule, von Curriculum und Studium", Felix Klein und die Berliner Schulkonferenz des Jahres 1900, Der Mathematikunterricht, 2000, 46: 3, S. 62-76.

Schubring, Gert: „L'Enseignement Mathématique and the First International Commission (IMUK): The Emergence of International Communication and Cooperation", One Hundred Years of L'Enseignement Mathématique. Moments of Mathematics Education in the Twentieth Century. Proceedings of the EM-ICMI Symposium Geneva, 20-22 October 2000, eds. Daniel Coray, F. Furinghetti, H. Gispert, B.H. Hodgson, G. Schubring. L'Enseignement Mathématique: Geneva, 2003, S. 47-65.

Tobies, Renate: „Zur wissenschaftsorganisatorischen Tätigkeit von Felix Klein im Rahmen der Breslauer Unterrichtskommission". NTM-Schriftenreihe für Geschichte der Naturwissenschaften, Technik und Medizin, 16 (1979) 2, S. 50-63.

Jürgen von Ungern-Sternberg/Wolfgang von UngernSternberg, Der Aufruf „An die Kulturwelt!“: das Manifest der 93 und die Anfänge der Kriegspropaganda im Ersten Weltkrieg; mit einer Dokumentation (Stuttgart: Steiner, 1996).

\section{Adresse des Autors \\ Dr. Gert Schubring \\ Universität Bielefeld \\ Institut für Didaktik der Mathematik \\ Postfach 100 । 31 \\ 3350I Bielefeld \\ gert.schubring@uni-bielefeld.de}

Gert Schubring, Jg. 44, Studium der Mathematik und Physik in Mainz und Bonn, Diplom in Mathematik, Promotion in Mathematik-Didaktik, Habilitation in Geschichte der Mathematik. Abteilungsleiter am Institut für Didaktik der Mathematik (U Bielefeld). Insbesondere aktiv in Wissenschaftler-Austausch-Projekten mit Brasilien.
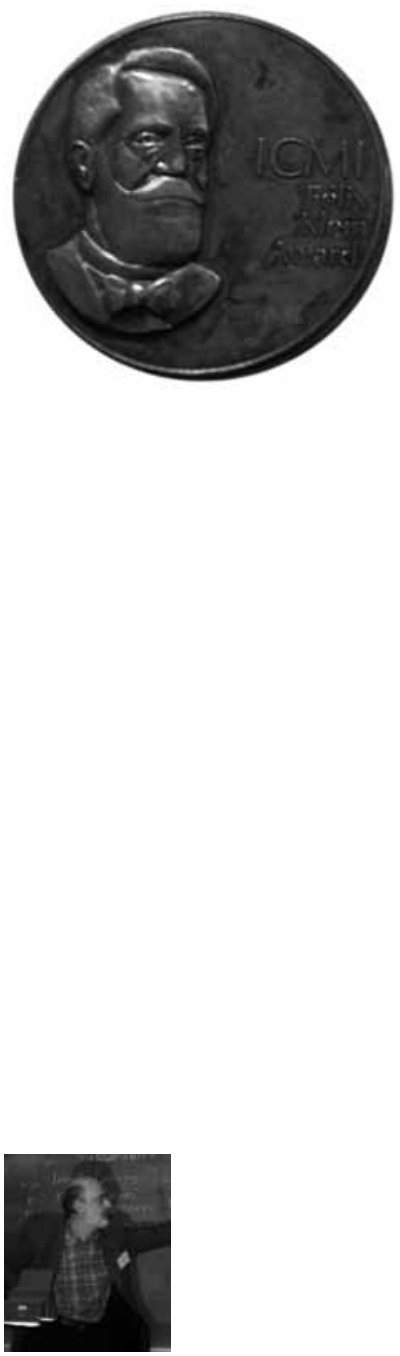